its study yields comprehensive results must be slow, because of the nature of its material and of the intricacy of the complex of physical and biotic factors within which all living organisms exist. It seems, therefore, that the Nature Conservancy may for some years be at best a one-eyed adviser, except where problems relate to only the most simple factors. It is relevant to emphasize that in the case of each of the four examples quoted in the first report no adequate answer could be returned. In fact, the reply was virtually, "We do not know, but we will try to find out", and research has been initiated in an effort to discover the solution.

To biologists therefore, as indeed to all who are prepared to take an objective view of conservation, the most interesting and stimulating sections of these two reports are those announcing the establishment and organization of the Conservancy's own research stations, at Merlewood, with Moorkouse at its doorstep, at Ananceun in the Beinn Eighe Reserve, and at Furzebrook in Dorset. Coupled with this must be Appendixes V, VI and VII to the second report, in which are listed the grants awarded to universities and other organizations for the development of research into specific field-problems. One of the strongest deterrents to the initiation of fundamental ecological research, particularly in relation to animal populations, has been the breadth of the task which must be faced. Even the so-called autecological study of the bionomics of a single species must involve the investigator in an infinitude of problems in which the physical factors of climate and microclimate, geology and soil structure and physiography may be involved, while willy-nilly an extensive study of the lives and relationships of a greater or lesser number of other species must be examined in order to determine the nature of their influence upon the species in which the worker is primarily interested. He must therefore be equipped in some measure as meteorologist, pedologist, taxonomist and statistician, and in a host of other techniques. Few men and women are able to attain to this width of interests, and in practice aid is sought from other experts in the solution of the many problems which have to be examined.

Synecology - the study of whole habitats and of the total assemblages of plants and animals within them-presents a still greater complexity of pattern, and demands so high a degree of concentration, knowledge and labour that few investigations of this lind have been successfully concluded. Nevertheless, the autecology of any species cannot be elucidated without reference to the synecology of the habitat in which it lives. It follows, therefore, that the ultimate aim of ecological science must involve a detailed synecological picture of at least the major habitats of the world. Only then will it become possible to assess correctly the status of the individual species; and, from the point of view of human welfare, only then will it become possible to prophesy the consequences to be expected from any attempt to modify the status of a species, whether by encouragement, control or, in the final event, extermination.

It seems logical to expect that comprehensive ecological knowledge can only be attained through the work of teams of experts in groups of related fields, and it is therefore encouraging to observe the nucleus of one such team being created in the scientific staff of the Nature Conservancy. It is to be hoped that, once the organization has escaped its growing pains, its scientists will be freed from all but the necessary minimum of administrative and advisory duties, in order that they may concentrate on research. Concurrently, the programme of assistance to universities and to reputable naturalist organizations is warmly welcome. There is a wealth of talent among amateur naturalists, about which they themselves are frequently unduly humble. The exact observer who is prepared to devote his leisure to the ordered study of an area, or of a group of animals or plants, can, and does, make as important contributions to biological knowledge as do many professional scientists. Indeed, the increased load of administrative and teaching duties which besets the university teacher is usually now so heavy as virtually to preclude him from spending sufficient time, at the right times, in the field. As a result, much of his ecological work must be done by proxy. It is here that the training and research grants made by the Conservancy to university departments are so important. Thus, not only is a continual stream of apprentice recruits provided, but also the experienced ecologist is enabled to stimulate and direct work for which he himself no longer has time or freedom.

In conclusion, it is relevant to refer to the 1947 report of the Wild Life Conservation Special Committe ${ }^{2}$. This committee examined the problem which has been adumbrated in the foregoing paragraphs, and made a number of recommendations, of which the most stimulating was perhaps that mooting the idea of freedom of movement of members of staff between museums, the Nature Conservancy and university departments. The ecological worker would thereby gain a wide experience of different disciplines, techniques and methods of attack upon his problems. Such increased versatility is so patently desirable as to create the hope that the idea has not been pigeon. holed and forgotten.

L. A. HARVEY

1 The Nature Conservancy. Reports of The Nature Conservancy for the period up to 30 th September 1952 . Pp. iii +33 . 18. 3d. net. Report of The Nature Conservancy for the Year ended 30th September 1953. Pp. iii +31, 18, $3 d$. net. (London: H.M. Stationery Offlce, 1953.)

- Conservation of Nature in England and Wales. Cmd. 7122. (London, 1947.)

\section{RECENT ADVANCES IN ANIMAL MORPHOGENESIS}

1 HE conference sponsored by the editorial board of the Journal of Embryology and Experimental Morphology and held in London during January 9-12 permitted a large number of biologists to hear and discuss accounts of recent work on many major aspects of animal morphogenesis. For this opportunity they were primarily indebted to their Continental colleagues, whose participation has provided an important stimulus to British interest in this field.

That rewarding work may still be found in the exploration of the classical problems of embryology was shown by Prof. J. Pasteels (Brussels), who described the first fruits of an investigation of embryonic stages of reptiles from the Belgian Congo. He has been able to show that the migration of the primordial germ cells to the gonadal ridges by a vascular route, long known in birds and Sphenodon, occurs also in lacertilians. A study of body form and brain morphology in species of different habits showed that relative functional importance in the adult can be anticipated by the embryo at a remarkably early stage. The brains of embryo 
chameleons (animals which in the adult rely largely upon their visual sense) and those of the embryo Mabuia (in the adult largely olfactory) show early differences in the size of optic and olfactory centres. Similar considerations seem to govern the form of the body generally and of the limb, both of which in adult lacertilians may show wide differences in form and function.

Although the general configuration of the embryo can show a precocious anticipation of the functional adaptations of the adult, things are not necessarily the same at the histological level. Prof. A. Stefanelli (Rome) described cells within the optic tectum of teleost fishes which are bipolar in form and possess a recurrent branch arising from the outer fibre. To see how far their differentiation was dependent upon function, studies were made of their development in oviparous fish ; in viviparous fish, in which animals before and after birth were compared ; and in blind cave fish compared with related forms with vision. Blind fish, in which the eyeball is normally nearly completely enclosed in a bony capsule and in which there is no functional optic nerve, may nevertheless possess some of these neurones with their major features intact. Blindness in this species seems to have led to a reduction in the number of neurones subserving vision without altering the general features of the histogenesis of those surviving.

Prof. F. E. Lehmann (Berne) has made an electron microscope study of the cytoplasmic constituents of blastomeres of known morphogenetic properties from the eggs of the oligochate Tubifex and of the sea-urchin. The technical problems of preparing the material have been overcome by treatment with glycerine or sucrose, which makes the cells brittle and easy to isolate from the germ and also dehydrates them sufficiently to inhibit autolysis. Fixation by osmium tetroxide provides good preservation of mitochondria, but a more acid fixative is required for the study of microsomes. The cells studied in the Tubifex germ were the somatoblasts $2 d$ and $4 d$, the first giving rise to ectoderm and the latter to mesoblast in normogenesis. The cytoplasm of these cells was shown to differ in the density of the population of mitochondria-4 $4 d$ having many more than $2 d-$ and in the nature and numbers of smaller inclusions. The microsome populations of different cells in the sea-urchin blastula also showed characteristic differences. In the micromeres are found many small microsomes, while there is a gradient in the concentration of mitochondria from animal to vegetal pole with a maximum at the animal pole.

Prof. Chr. P. Raven (Utrecht) gave an account of the effects of lithium ions on the development of Limnaea stagnalis, and of some ways in which these effects could be modified. Treatment with lithium chloride may induce exogastrulation and cyclocephalic malformations - in extreme cases cyclopia itself. These two effects are, however, independent, and it is suggested that the effect of lithium is both to weaken an animal field and to disturb the vegetal pole material. There is evidence that exogestrulation and cyclopia are independent consequences of a causal chain in which the lithium has a primary effect mediating a secondary disturbance of the respiratory processes which is responsible for the observed tertiary effects. Combinations of other ions with lithium show that while potassium, if anything, inereases the lithium effect, either calcium or potassium cyanide may exercise \& protective function. Conditions of oxygen lack, which in themselves induce exogastrulation, are not so effective in presence of lithium ions. Concentrated potassium cyanide and heat shocks may both imitate the lithium effect. Dilute potassium cyanide under conditions of anaerobiosis increases the effectiveness of the latter as an agent causing exogastrulation. Exogastrulation, but not cyclopia, can be produced by centrifugation.

The mammalian egg, still largely inaccessible to the surgical procedures used on lower vertebrates, is coming to assume its rightful place in contemporary embryological advance by the application to it of the methods of cytochemistry, and Prof. A. Dalcq (Brussels) showed how great the recent increase in our knowledge of its biochemistry has been. Studies on mitochondria and other visible particles in the maturing oocyte during fertilization and the early stages of development have been combined with examinations of the distribution of nucleic acids, polysaccharides and alkaline phosphatase. During oogenesis the most important changes seem to concern the microsomes and larger visible granules, these being active in the synthesis of cytoplasm and metaplasmic inclusions. Nuclear extrusions are also important at this stage. Fertilization sees little change in the visible cytoplasmic particles; but nuclear activation is accompanied by a temporary reduction in the deoxyribonucleic acid of both sperm and oocyte nuclei. The deoxyribonucleic acid is, however, rapidly recovered as the pronuclei form. Early development sees the first appearance of alkaline phophatase. Bilateral symmetry, already apparent in the distribution of cell constituents in the maturing oocyte, becomes marked in the later cleavage stages, and from this time onward differences in the cytoplasmic constitution of cells with different fates become apparent. To some extent it is already possible to explain the biochemical activity of these cells in terms of their capacity for performing certain morphogenetic functions.

Prof. S. Hörstadius (Úppsala) described a further analysis of the organization of the young sea-urchin germ. The reduction of Janus green in cells under conditions of diminishing oxygen tension can be used to distinguish between regions of the egg with high and those with low metabolic activity. In such conditions a normal egg will show reduction (that is, high metabolic activity) first at the vegetal pole, then in the apical region and last of all in the region of the ciliary band. Isolated animal and vegetal halves in these conditions display simultaneous onset of Janus green reduction, and this indicates that the vegetal region normally inhibits the apical one. Micromeres implanted into the side of the animal hemisphere induce a reduction centre there, and are also effective in distorting the gradient in mitochondrial density that runs from animal to vegetal poles. Work with anti-metabolites had revealed a rather surprising phenomenon, since while 8-chloroxanthene (blocking purine and nucleic acid metabolism) had an animalizing effect and phenyllactic acid (blocking amino-acid metabolism) had a vegetalizing effect, both gave rise to a number of peculiar radially symmetrical larvæ of a kind that Gustafson had produced with detergents. Both substances were, in fact, acting in this way, though it is open to question how far this is relevant to their other morphogenetic effects.

In the classical experiments on neurel induction in the urodele embryo the structures induced were either ectodermal in nature or associated with ectodermal inductions. It has now proved possible to 
induce structures of wholly, or almost wholly, mesodermal nature. Prof. S. Toivonen (Helsinki) reported a study of inductions produced by alcoholtreated guinea-pig bone marrow after its implantation into the gastrula or when placed in ectodermal 'sandwich' explants. It is noteworthy that the tissues induced in such experiments are those which in normogenesis would arise from both endomesoderm and ectomesoderm. Secondary inductions sometimes, but by no means always, occur. The same inductor if further treated with hot water for a short period undergoes a remarkable change in properties. It now induces only structures arising in normogenesis from the ectoderm, for example, lenses and balancers, though rarely, if ever, neural cells. Prof. Toivonen believes that this change is not due to a transformation of the mesoderm inductor but results from its complete destruction, with the consequent unmasking of an ectodermal inductor that had previously been unable to express itself.

Prof. J. Brachet (Brussels) described a technique for the autoradiographic localization of labelled atoms developed in his laboratory by Mme. A. Ficq. It uses the thick emulsions prepared for the study of cosmic radiation. The method permits of the location of labelled atoms with a resolution of about 1 micron. Experiments on the oocytes of Asterias using marked glycine showed that the activity of the nucleolus was nearly a hundred times as great as that of either nuclear sap or cytoplasm after one hour's treatment. When applied to the process of embryonic induction the method revealed that when either labelled glycine or orotic acid is incorporated into an amphibian organizer that is then implanted into another gastrula, radioactivity can later be detected not only in the secondary neural tube but also, to a lesser extent, in the host's normal nervous system. The method can also be used to identify lithium if the preparations are subjected to neutron bombardment which splits the lithium atoms. It has been possible in this way to demonstrate a distinct accumulation of lithium in the dorsal half of amphibian gastrulæ formed from eggs treated with 1 per cent lithium. chloride.

Prof. C. H. Waddington (Edinburgh) reported the methods being developed in his laboratory using radioactive amino-acids and the stripping film method in the study of embryonic processes in amphibians and birds. Using radioactive methionine and glycine, it had proved possible to get usable concentrations in axolotl and Xenopuss embryos, and radioactive methionine could successfully be introduced into chick embryos by injecting the albumen in the neighbourhood of the blastoderm and maintaining the egg at room temperature for 12-24 hr. Preliminary resalts suggested that significant differences in the subsequent radioactivity of different organ rudiments could be detected.

From Prof. Waddington's laboratory there also came a film, exhibited by Dr. D. A. Ede, of the development of Drosophila. A surprising amount of the morphogenetic activity from fertilization onwards can be seen by time-lapse cinematography, of the whole egg, and shots of eggs doomed to early death by their genetic constitution showed that the method holds great promise for the study of abnormal development.

For workers on the Protozoa, cell division does not serve morphogenetic processes so much as itself set the scene in which morphogenetic processes must necessarily play their part. Prof. E. Fauré-Fremiet
(Paris) described some of the processes which accompany the division of the ciliate Urocentrum turbo into an anterior individual or proter and a posterior one or opisthe. In this species the kinetosomes of the meridional kineties are so arranged as to lie in circular transverse lines ending in an anterior belt or fascia and an equatorial cingula. At the onset of division the cingula widens by the multiplication of its kinetosomes. It is thus a morphogenetic zone corresponding to Calkins's division zone. The cingular kinetosomes then become distributed along the meridional somatic kineties to complete the morphogenesis of the anterior part of the opisthe and the posterior part of the proter. The oral ciliation presents an autonomous systern with its own morphogenetic zone.

Prof. E. C. Wolff (Strasbourg) described the progress made in his laboratory in the attempt to replace chick embryo extract by known mixtures of amino. acids as a medium permitting the survival, differentiation and growth of rudimentary organs in vitro. Using the syrinxes, tibias or the gonads taken from bird embryos of 7-9 days incubation as explants, media composed of agar, glucose and saline to which amino-acids and vitamins had been added were tested for their power to sustain growth and differentiation over a seven-day culture period. Growth in the explants was judged by the presence of mitoses and more particularly by the increase in total nitrogen. Marked growth and differentiation in media containing many amino-acids had been obtained; but when an attempt was made to find the minimum requirements of amino-acids for the explants it wos discovered that though growth can occur in media to which only a few had been added, the requirements of different organ-rudiments are not the same.

Mr. M. Abercrombie (London) described observations on chick heart fibroblasts in tissue culture which had suggested that 'contact inhibition' was an important factor determining the behaviour of the individual cells and some of the properties of the whole culture. By 'contact inhibition' is meant inhibition of moverment, in directions normal to the plane of contact, in two contiguous cells. The evidence for the existence of this phenomenon comes mainly from the behaviour of the outgrowths from two neighbouring explarits at their zone of junction in a fluid medium. The failure of the outgrowths to 'overlap' or be thrown into folds bears striking testimony to the unwillingness of their cells to climb over one another. Contect inhibition is not only consistent with well-known features of fibroblast cultures, but may actually explain many of them. Interactions between outgrowths of explants from different organs, and even from different species, go to confirm the generality of the phenomenon, while the three-dimensional outgrowths from explants in plasma clots show it in conditions more nearly comparable with the situation in which fibroblasts occur in the organism.

A number of the papers were accompanied by demonstrations of research material, and in addition such material was presented by Dr. S. M. Manton and Mr. K. T. Anderson illustrating maternal-embry. onal connexions in viviparous Onychophora, by Prof. P. B. Medawar, Dr. R. E. Billingham and Mr. L. Brent to illustrate the principle of actively acquired tolerance of foreign cells in mammals and birds, and by Dr. D. R. Newth on the behaviour of lamprey neural crest cells in homoplastic and xenoplastio transplantation. 
Dr. R. A. Beatty, Dr. M. Fischberg, Dr. J. Needham, Dr. Muriel Robertson, Prof. J. Z. Young and a number of others contributed to the discussions on the papers read.

A most valuable discussion on the nature of inducing stimuli, led by Prof. J. Pasteels, was also held. It showed that interest in this subject is still high, and that the seemingly formidable difficulties that blocked the search for a single chemical or physical agent of neural induction have not prevented the accumulation of ever more precise information on the biology of the process.

The conference was made possible by the generosity of the Royal Society, the Ciba Foundation, the British Council and the Wellcome Foundation.

D. R. NEWTH

\section{METALLIC TRANSFER IN SCREWING AND ITS SIGNIFICANCE IN BONE SURGERY}

\section{By DR. F. P. BOWDEN, F.R.S., and J. B. P.} WILLIAMSON

Physics and Chemistry of Surfaces, Department of Physical Chemistry, University of Cambridge and P. GOWANS LAING

The Royal Infirmary, Bradford

GARLIER experiments have shown that when two metals are placed in contact, appreciable metallic transfer occurs from one surface to the other. If sliding takes place, the amount of metal transferred is increased considerably. A general account of this work has been given elsewhere ${ }^{1}$.

In simple mechanical operations such as screwing or bolting, we may expect that some transfer will occur between the screwdriver and the screw or the spanner and the bolt. Although the transfer will be small, it may under certain conditions be important. For example, in modern surgery it is customary to use metallic plates to strengthen or to repair a fractured bone. These are screwed or bolted on to the bone and may remain permanently in position. The plates, screws and bolts are all made from a stainless steel which has a very high corrosion resistance. Intensive investigations have been undertaken in recent years to select the best steel for this purpose. It has been found that steel of the specification shown in Table $l(a)$ is one of the most inert when in contact with human tissues ${ }^{2}$. It was also found that if metal alloys of differing compositions are placed in contact in the human body, metal corrosion and 'electrolytic inflammation' in the tissues occur'. 'Electrolytic inflammation' consists in the inflammatory cells of the tissues becoming more numerous and an increased local vascularity of bone and soft tissue. This condition interferes with the normal healing of bone and soft tissues and may eventually produce heavy scarring. Orthopædic surgeons therefore take great care that all plates and screws which are to be left in contract are made of the same alloy. The insertion of 'mixed motals' is always avoided. These plates and screws are electrolytically polished and are passivated in nitric acid. Nevertheless, it is found in practice that inflammation and corrosion do occasionally occur.

Table 1

(a)

Specification for screws and plates

$\begin{array}{lr}\text { (per cent by weight) } \\ \text { Carbon } & 0.07 \\ \text { Silicon } & 0.30 \\ \text { Manganese } & 0.5 \\ \text { Chromium } & 18.0 \\ \text { Nickel } & 8.0 \\ \text { Iron } & 70.38 \\ \text { Molybdenum } & 2.75\end{array}$

The screwdrivers, spanners and forceps used in this form of bone surgery are also made from stainless steel, but of a composition differing from that of the plates (see Table $I b$ ) and are less resistant to corrosion.

If small fragments of this different steel are welded on to the screws during the screwing operation, they may, when immersed in the body tissue, form small electrolytic couples which cause inflammation and accelerate corrosion. Experiments were therefore carried out to investigate whether metallic transfer occurred and to determine its amount and distribution. Measurements showed that the steel for the tools was appreciably softer (V.P.H. 150) than the steel for the screws and plates (V.P.H. 380).

Experimental. The experiments consisted of driving the screws with a screwdriver having a radioactive tip, and then determining the amounts of metal transferred from the driver to the screw heads by measuring their acquired radioactivities. In order that the forces involved should approximate as closely as possible to those occurring in an actual operation, the screws were driven into bone. The expeziment was carried out using samples of splints and self-tapping screws manufactured for medical use, and a splint was fastened by four screws to a leg bone taken from fresh beef. The screws were started by drilling a small hole in the bone with an electric hand-drill and were then driven home and tightened with a screwdriver. This driver had a normal handle and shank, but, instead of a tip, had a small hole recessed into its end. Into this hole a slab of metal $\frac{1}{16}$ in. $\times \frac{1}{4}$ in. $\times \frac{1}{2}$ in. cut from the tip of an actual medical screwdriver was inserted and used as the screwdriver tip.

Before the experiment, this slab was irradiated in the neutron pile at Harwell and acquired $\&$ radioactivity of about $\frac{3}{4}$ me. For the present purpose only the activity of the chromium in the steel was significant. This consists of $K$-radiation and $\gamma$-radiation of $0.323 \mathrm{MeV}$. The screws and the driver were degreased with suitable solvents and the first screw was driven home firmly but carefully, so as not to cause appreciable slip between the driver and head. The driver slipped slightly in the slot while the second screw was being driven in; and two deliberate slips were made along the slot of the third screw. The fourth screw was driven and tightened with the utmost caution, and no slip was perceptible.

A Geiger counter was used for measuring the acquired activity of each head. Only $\gamma$-rays were counted. Thus any radiation emanating from transferred metal adhering to the bottom of the slot was not shielded by the mass of the screw head, and the entire amount of adherent metel was recorded.

Results. The specific strength of the radioactive metal was measured by counting the $\gamma$-emission from the slab used as the screwdriver tip, in the same Geiger counter under the same geometrical conditions; the weight of this slab having been determined before irradiation. In this way the mass of metal trans. 